\title{
ARTICLE OPEN \\ Strain-mediated voltage-controlled switching of magnetic skyrmions in nanostructures
}

\author{
Jia-Mian $\mathrm{Hu}^{1,2}$, Tiannan Yang ${ }^{1}$ and Long-Qing Chen ${ }^{1}$
}

\begin{abstract}
Magnetic skyrmions are swirling spin structures stabilized typically by the Dyzaloshinskii-Moriya interaction. The existing control of magnetic skyrmions has often relied on the use of an electric current, which may cause overheating in densely packed devices. Here we demonstrate, using phase-field simulations, that an isolated Néel skyrmion in a magnetic nanodisk can be repeatedly created and deleted by voltage-induced strains from a juxtaposed piezoelectric. Such a skyrmion switching is non-volatile, and consumes only $\sim 0.5 \mathrm{fJ}$ per switching which is about five orders of magnitude smaller than that by current-induced spin-transfertorques. It is found that the strain-mediated skyrmion creation occurs through an intermediate vortex-like spin structure, and that the skyrmion deletion occurs though a homogenous shrinkage during which the Néel wall is temporarily transformed to a vortexwall. These findings are expected to stimulate experimental research into strain-mediated voltage control of skyrmions, as well as other chiral spin structures for low-power spintronics.
\end{abstract}

npj Computational Materials (2018)4:62 ; doi:10.1038/s41524-018-0119-2

\section{INTRODUCTION}

Magnetic skyrmions typically exhibit a cycloidal or helical spin rotation along the radial plane from one perpendicular direction to the other, known as a Néel Skyrmion or a Bloch skyrmion, respectively. The topological charge number ${ }^{1} Q=(1 / 4 \pi) \int \mathbf{m}$. $\left(\partial_{x} \mathbf{m} \times \partial_{y} \mathbf{m}\right) d x d y$ (where $\mathbf{m}$ denotes the normalized local magnetization) has a magnitude of about 1 in both types of skyrmions, 0.5 in a magnetic vortex, and 0 in a ferromagnetic state. Nanoscale skyrmions in magnetic materials are typically stabilized by Dyzaloshinskii-Moriya interaction (DMI), which arises either intrinsically within a bulk lattice or due to the proximity to an adjacent heavy-metal (Fig. 1a). ${ }^{2-4}$

The ability to use external stimuli to control (e.g., switch, move) a magnetic skyrmion underpins its potential application in spintronics, such as a skyrmion-based magnetic random access memory ${ }^{5}$ or a race-track memory. ${ }^{6}$ Various stimuli have been used, e.g., magnetic fields, ${ }^{7,8}$ electric currents, ${ }^{6,9-17}$ laser, $^{18,19}$ voltages, $^{20-}$ ${ }^{23}$ and stress. ${ }^{24-30}$ Among them, using voltages or strains are particularly promising because the heat dissipation in both schemes is negligible. Notably, repeated skyrmion creation and deletion induced by voltages 21,22 or stress ${ }^{25,30}$ have been demonstrated. However, these reports ${ }^{21,22,25,30}$ all involve a simultaneous application of a bias magnetic field to stabilize the skyrmion, which would hamper the device miniaturization because it is challenging to localize a magnetic field on a chip.

Here we propose an architecture that integrates a multilayer magnetic nanostructure with an underlying piezoelectric layer, where the former consists of a magnetic ultrathin nanodisk sandwiched by a capping layer and a heavy-metal underlayer (Fig. $1 b)$. In this architecture, we computationally demonstrate a repeated creation and deletion of an isolated Néel skyrmion by voltage-induced strains without using any magnetic fields. Note that recent experimental observations have demonstrated that an isolated magnetic skyrmion can be stable in the absence of magnetic fields in a magnetic nanostructure with a 3D geometric confinement. ${ }^{31,32}$ Here we limit our discussion to the case where a net DMI arises only from the magnet/heavy-metal interface. In this case, the formation of a Néel skyrmion is more favorable than a Bloch skyrmion, which can be seen from the expression of the interfacial DMI free energy (Eq. 4 in Methods). Figure 1c schematically shows the two degenerate spin structures of an isolated Néel skyrmion with downward $(Q=-1)$ and upward $(Q$ $=1$ ) cores, assuming the effective interfacial DMl strength $D$ in Eq. (4) is positive.

Using a 1.1-nm-thick $\mathrm{Co}_{20} \mathrm{Fe}_{60} \mathrm{~B}_{20}$ (CoFeB) nanodisk as an example, we computationally identified the ranges of disk diameter and $D$ where an isolated Néel skyrmion is stable or metastable. Upon applying a voltage across the piezoelectric layer (Fig. 1b), the induced strain can be transmitted to the CoFeB disk and modulate its magnetic free energy via magnetoelastic coupling ${ }^{33-37}$ and possibly the $D$. Our simulations then show that, if (1) a ferromagnetic state and a Néel skyrmion are stable or metastable under zero magnetic fields and (2) the gap between the energy levels of these two states is not too large, the voltageinduced strain alone can drive a non-volatile switching from the ferromagnetic state to the skyrmion (creation) and vice versa (deletion).

\section{RESULTS}

Stability and size of an isolated Néel skyrmion in a magnetic nanodisk

Figure 2a shows the computed stability diagram of three types of spin structures under different disk diameters and $D$. These three structures, represented by differently line patterns, include an isolated Néel Skyrmion, a quasi single domain (QSD, a

\footnotetext{
${ }^{1}$ Department of Materials Science and Engineering, The Pennsylvania State University, University Park, PA 16802, USA and ${ }^{2}$ Department of Materials Science and Engineering, University of Wisconsin-Madison, Madison, WI 53706, USA

Correspondence: J-M. Hu (jhu238@wisc.edu) or L-Q. Chen (Iqc3@psu.edu)
}

Received: 3 August 2018 Accepted: 22 October 2018

Published online: 21 November 2018 
a

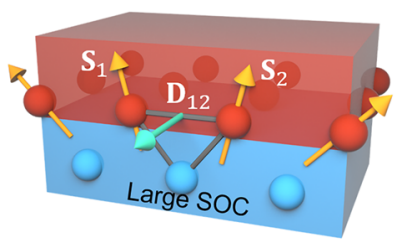

b

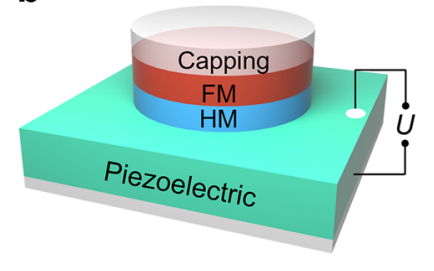

C

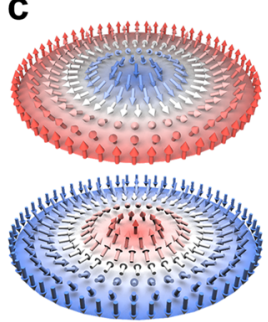

Fig. 1 Architecture. a, Schematic of the interfacial DMI in juxtaposed magnetic and heavy-metal layers. $\mathbf{S}_{1}$ and $\mathbf{S}_{2}$ denote the spins of two neighboring atoms in the magnetic layer that are exchange coupled through an atom of large spin-orbit coupling (SOC) in an adjacent heavymetal layer. $\mathbf{D}_{12}$ is the DM vector which is parallel to the interface and perpendicular to the triangle of the three atoms. $\mathbf{b}$, Heterostructures for strain-mediated voltage $(U)$-controlled switching of magnetic skyrmions: (from top to bottom) a capping layer; a ferromagnetic (FM) nanodisk with perpendicular magnetic anisotropy (PMA) and robust magnetoelastic coupling; a heavy-metal (HM) underlayer; a piezoelectric layer; an electrode. The capping layer can be a non-magnetic oxide or a dissimilar heavy metal to enhance the PMA and/or the interfacial DMI strength. c, Schematics of two degenerate Néel skyrmions induced by interfacial DMI with a positive average strength $D$

ferromagnetic state with titled spins near edges), and a stripe domain with Néel walls; see exemplary structures alongside the diagram. In areas where three line patterns overlap, all three spin structures can exist at equilibrium: the structure having the lowest intrinsic free energy density $f_{\text {intrin }}$ (see definition in Methods) is considered as thermodynamically stable while the other two metastable. Areas where two line patterns overlap can likewise be understood. Stable QSD, skyrmion, and stripe are colored blue, green, and yellow, respectively. For example, a skyrmion can exist at equilibrium wherever the three line patterns overlap, but it is thermodynamically stable only in the green area (i.e., relatively large disk diameter together with an intermediate $D$ ). Notably, a stable Néel skyrmion is always accompanied by two other metastable spin structures (i.e., all the green area hosts three line patterns) and generally only shows a slightly smaller $f_{\text {intrin }}$ than the stripe domain and/or the QSD (see top panels of Fig. 2b-c).

The size of an isolated Néel skyrmion is also strongly influenced by the $D$ and the magnetic disk diameter. As shown in Fig. 2b, under the same disk diameter of $220 \mathrm{~nm}$, the skyrmion diameter $\left(d_{\mathrm{Sk}}\right.$, defined as the diameter of the line $\left.m_{\mathrm{z}}=0^{6}\right)$ increases nonlinearly from about $40 \mathrm{~nm}$ to $150 \mathrm{~nm}$ upon increasing $D$ from $0.4 \mathrm{~mJ} / \mathrm{m}^{2}$ to $3 \mathrm{~mJ} / \mathrm{m}^{2}$. Such a nonlinear feature becomes appreciable when $D$ is larger than a critical value $D_{\mathrm{c}}$, which was analytically estimated (see Supplemental Note S1) to be about $0.7 \mathrm{~mJ} / \mathrm{m}^{2}$ for the 220 -nm-diameter disk (marked by broken line). Similar nonlinear features also appear in the cases of other disk diameters that have different $D_{c}$ (see Supplemental Fig. S1a-b). These nonlinear features appear because the edge confinement tends to suppress the growth of a skyrmion when $D>D_{\text {c. }}{ }^{38}$

Furthermore, when the disk diameter increases from $80 \mathrm{~nm}$ to $720 \mathrm{~nm}$ at $D=0.75 \mathrm{~mJ} / \mathrm{m}^{2}, d_{\mathrm{sk}}$ increases almost linearly from about $16 \mathrm{~nm}$ to $500 \mathrm{~nm}$ (see Fig. 2c). This indicates a nontrivial role of edge confinement on the $d_{\mathrm{Sk}}$ in the range of disk diameters investigated, because $d_{\mathrm{Sk}}$ would otherwise remain the same whether the hosting magnetic disk is large or small. ${ }^{38}$ As $d_{\mathrm{Sk}}$ increases, the feature of the local magnetization profile changes from a compact-skyrmion-type (where the Néel wall thickness is similar to $d_{\mathrm{Sk}}$ ) to a bubble-type (Néel wall thickness \&mle; $d_{\mathrm{Sk}}$ ), as shown in Fig. S1c-d. Note that the stability of such an isolated skyrmion reduces when the disk diameter exceeds $300 \mathrm{~nm}$, evidenced by the associated slight increase of the $f_{\text {intrin }}$ (top panel of Fig. 2c). The value of $300 \mathrm{~nm}$ is close to the analytically calculated spin cycloid period $L_{0}=2 \pi\left(2 A_{\mathrm{ex}} / D\right.$ ) (where $A_{\mathrm{ex}}$ is the Heisenberg exchange coefficient), about $318 \mathrm{~nm}$ for $D=0.75 \mathrm{~mJ} /$ $\mathrm{m}^{2}$. Associated with the reduced stability of one isolated Néel skyrmion at large disk diameters, the formation of multiple isolated Néel skyrmions in the same disk becomes more favorable (see Supplemental Fig. S2). In fact, multiple isolated Néel skyrmions have been experimentally observed in a $2-\mu \mathrm{m}$-diameter $\mathrm{Pt} / \mathrm{Co} / \mathrm{Ta}$ disk at zero magnetic fields. ${ }^{13}$ Roughly speaking, one isolated Néel skyrmion in a disk can be stable if the disk diameter is not significantly larger than $L_{0}{ }^{38}$

Strain-mediated creation and deletion of one isolated skyrmion Using a 220-nm-diameter CoFeB disk with $D=0.75 \mathrm{~mJ} / \mathrm{m}^{2}$ as an example, we show that switching of an initial QSD to a skyrmion (creation) requires the application of a minimum biaxial in-plane isotropic tensile strain $\varepsilon_{\text {app }}$ of about $0.4 \%$ (see inset of Fig. 3a). The initial QSD state was obtained after the application and subsequent removal of a large perpendicular magnetic field. The temporal evolution of $\Delta f_{\text {intrin }}$ (the bottom of Fig. 3a) shows that the skyrmion creation occurs through a dynamically oscillating process, going through multiple transitional states. Here the transitional states are defined as where the $\Delta f_{\text {intrin }}$ peaks during the oscillation. The time when $\Delta f_{\text {intrin }}$ reaches its highest peak $\left(\sim 34.5 \mathrm{~kJ} / \mathrm{m}^{3}\right)$ is denoted as $t_{\mathrm{ts}}$. At $t=t_{\mathrm{ts}}$, a vortex-like spin structure appears (Fig. 3b), corresponding to a $Q$ value of about 0.4 (note that $|Q|=0.5$ in an ideal in-plane vortex). Spin structures at the initial ( $t=0 \mathrm{~ns}$ ) and the equilibrium states $\left(t_{\text {eq }}=22.37 \mathrm{~ns}\right.$, see definition in Methods) are also shown in Fig. 3b, while a complete skyrmion creation process is shown in Supplemental Video S1. The thermodynamic condition for passing the highest transitional state $\left(t=t_{\mathrm{ts}}\right)$ is written as,

$\Delta f_{\text {tot }}\left(t_{\text {ts }}\right)=\Delta f_{\text {intrin }}\left(t_{\text {ts }}\right)+\Delta f_{\text {elast }}\left(t_{\text {ts }}\right)<0$.

When $\varepsilon_{\mathrm{app}}=0.4 \%, \Delta f_{\text {elast }}\left(t_{\mathrm{ts}}\right)$ is about $-35.7 \mathrm{~kJ} / \mathrm{m}^{3}$, yielding a negative $\Delta f_{\text {tot }}\left(t_{\mathrm{ts}}\right)$. Temporal evolution of the $\Delta f_{\text {elast }}$ (the change of elastic energy density), as well as other energy densities contributing to the $\Delta f_{\text {intrin }}$ are shown in Supplemental Fig. S3a, which shows that the skyrmion creation is driven by the minimization of elastic, stray field, and interfacial DMl energy at the expense of increasing the exchange and anisotropy energy. As shown in Fig. 3b, an upward-core Néel skyrmion $(Q \approx 1)$ appears at equilibrium. However, a degenerate downward-core skyrmion $(Q \approx-1)$ can appear under different $\varepsilon_{\mathrm{app}}$ and $D$ (see Fig. S3b-c and analyses therein).

Our simulations also show that, starting from the same QSD state, the equilibrium spin structure would be a vortex-like spin structure $(|Q|$ reduces toward 0.5$)$ instead of the desirable Néel skyrmion when $\varepsilon_{\mathrm{app}}$ is relatively large (see Supplemental Fig. S4). In this case, more spins would lie within the horizontal plane to minimize the elastic energy and meanwhile form a vortex to minimize the stray field energy, at the expense of increasing the interfacial DMI energy.

We further found that a minimum biaxial in-plane isotropic compressive $\varepsilon_{\text {app }}$ of about $-0.24 \%$ (see inset of Fig. $4 \mathrm{a}$ ) is required to switch a skyrmion to a QSD state (deletion) in a 220-nmdiameter CoFeB disk with $D=0.75 \mathrm{~mJ} / \mathrm{m}^{2}$. The initial skyrmion structure $(t=0$, shown in Fig. $4 b)$ was obtained by evolving the 
a
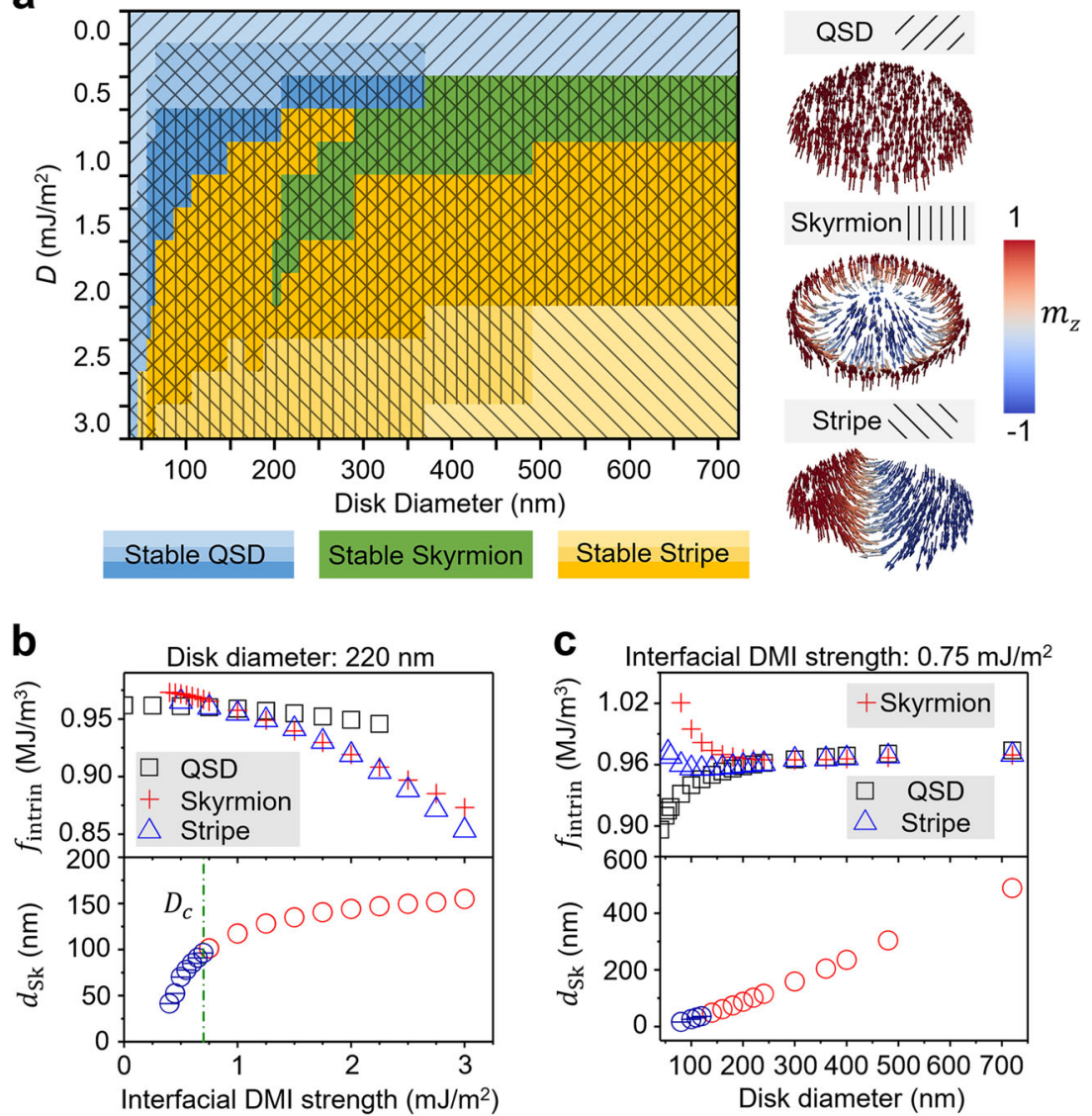

Fig. 2 Thermodynamic stability diagram. a, Stability diagram of the quasi single domain (QSD), Néel skyrmion, and Néel stripe (represented by different line patterns) as a function of the CoFeB disk diameter and the interfacial DMI strength $D$. Typical spin structures are shown on the right. Areas of stable (i.e., ground-state) QSD, skyrmion, and stripe are colored blue, green, and yellow, respectively. Areas with overlapping line patterns indicate the existence of one or two metastable spin structures in addition to the stable spin structure. Color bar $m_{z}$ denotes local normalized perpendicular magnetization. Dependence of the intrinsic magnetic free energy density $f_{\text {intrin }}$ and the skyrmion diameter $d_{\mathrm{sk}}$ on $\mathbf{b}$, the interfacial DMI strength $D$, and c, the CoFeB disk diameter. The disk diameter is $220 \mathrm{~nm}$ for all cases in $\mathbf{b}$, while the $D$ is $0.75 \mathrm{~mJ} / \mathrm{m}^{2}$ for all cases in c. When $D<0.75 \mathrm{~mJ} / \mathrm{m}^{2}$ in $\mathbf{b}$ and when disk diameter $<140 \mathrm{~nm}$ in c (marked by blue crossed circles), the skyrmion exhibits a notably higher energy level than the other spin structures. These highly metastable skyrmions appear at equilibrium in simulations only when the inplane size of a simulation cell is relatively small (i.e., $0.5 \mathrm{~nm}$ ). The vertical line in $\mathbf{b}$ marks the critical interfacial DMl strength $D_{\mathrm{c}}\left(\approx 0.7 \mathrm{~mJ} / \mathrm{m}^{2}\right)$ for a 220-nm-diameter 1.1-nm-thick CoFeB disk

skyrmion at $t=t_{\text {eq }}$ (Fig. $3 \mathrm{~b}$ ) to equilibrium under zero strain. Compared to the multiple transitional states in the case of skyrmion creation, only two appreciable transitional states need to be passed (see the evolution of $\Delta f_{\text {intrin }}$ in Fig. 4a). The $\Delta f_{\text {intrin }}$ at the highest transitional state (where $t=t_{\mathrm{ts}}=1.22 \mathrm{~ns}$ ) is about $5.25 \mathrm{~kJ} /$ $\mathrm{m}^{3}$, while the $\Delta f_{\text {elast }}\left(t_{\mathrm{ts}}\right)$ is about $-7.63 \mathrm{~kJ} / \mathrm{m}^{3}$. Based on Eq. (1), $\Delta f_{\text {tot }}\left(t_{\mathrm{ts}}\right)$ is negative, ensuring the pass of highest transitional state. Analyses on the individual evolution profiles of contributing energy terms (similarly to Fig. S3a) show that the strain-mediated skyrmion deletion is driven by the minimization of elastic, exchange, and anisotropy energy, at the expense of increasing the interfacial DMI and stray field energy. Spin structures at the highest transitional state $\left(t=t_{\mathrm{ts}}\right)$ and upon the completion of switching $\left(t=t_{\mathrm{sw}}=1.63 \mathrm{~ns}\right)$ are also shown in Fig. 4b. Here the $t_{\mathrm{sw}}$ is defined as the time when the skyrmion diameter reduces to zero. A complete skyrmion deletion process is shown in Supplemental Video S2. Figure $4 \mathrm{~b}$ along with the video demonstrates that the skyrmion deletion occurs through a homogeneous shrinkage, during which the Néel wall is temporarily transformed into a vortex wall to minimize the stray field energy. Such a vortex wall can be seen in the spin structure at $t=$ $t_{\mathrm{ts}}$ and more clearly in the video.

Based on the mechanisms discussed above, we have further simulated a non-volatile and reversible switching between a skyrmion and a QSD (i.e., repeated skyrmion creation and deletion) using pulses of bipolar in-plane isotropic strains (Supplemental Fig. S5). The minimum duration of the tensile strain pulse for creating the skyrmion is found to be the time when $Q$ increases to about 1 for the first time ( $3 \mathrm{~ns}$, see Fig. 3a). The minimum duration of the compressive strain pulse equals $t_{\mathrm{sw}}$.

\section{DISCUSSION}

The top panel of Fig. 5a shows the critical strain for skyrmion creation as a function of $D$ under the same disk diameter of $220 \mathrm{~nm}$. There are two main messages. First, there exists a range of $D\left(0.75 \sim 1.75 \mathrm{~mJ} / \mathrm{m}^{2}\right)$ only within which a skyrmion can be created from an initial QSD. When $D$ is relatively small $\left(0.4 \sim 0.5 \mathrm{~mJ} / \mathrm{m}^{2}\right)$ where the skyrmion state has an appreciably higher energy level than the other states (Fig. 2b), the equilibrium spin structure resembles an in-plane magnetic vortex (see Supplemental Fig. S6). This is because the switching is dominated by the minimization of elastic energy, similarly to the cases when $\varepsilon_{\text {app }}$ is relatively large (Fig. S4). When $D$ is relatively large $\left(2-2.25 \mathrm{~mJ} / \mathrm{m}^{2}\right)$ where the Néel stripe has a lower energy level than the skyrmion (Fig. 2b), the equilibrium spin structures would then be a Néel stripe (also see Fig. S6). This is because at large $D$, the minimization of interfacial DMI free energy plays a more important role in the overall process 

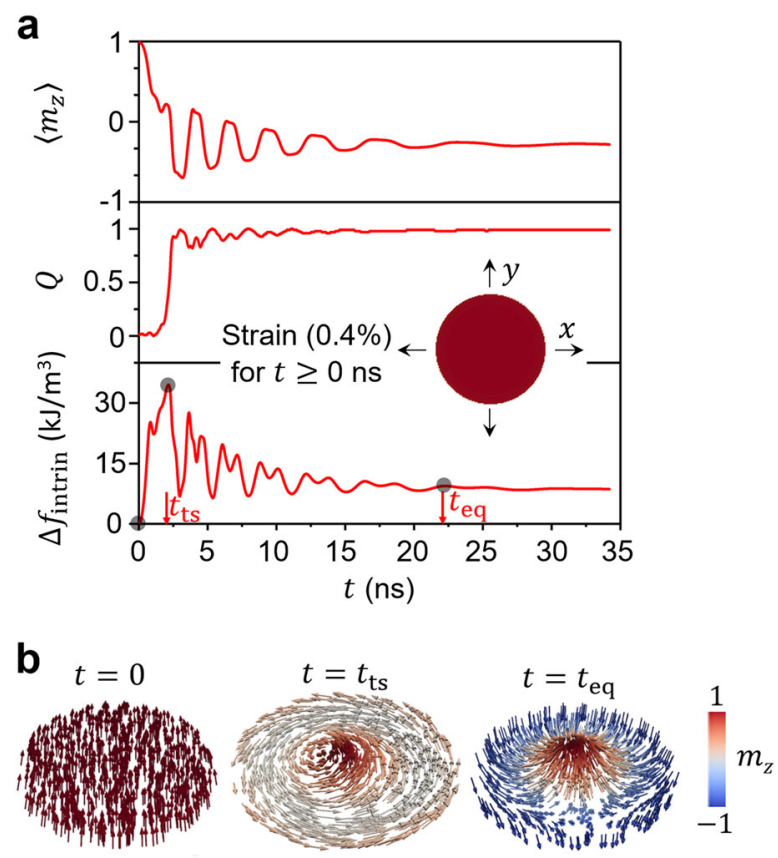

Fig. 3 Skyrmion creation. a, Temporal evolution of the $m_{z}$ (volume average of the normalized perpendicular magnetization), $Q$ (the topological charge number), and the change of the intrinsic magnetic free energy density $\Delta f_{\text {intrin }}(t)=f_{\text {intrin }}(t)-f_{\text {intrin }}(t=0)$ when a biaxial in-plane isotropic $\left(\varepsilon_{\mathrm{app}}=\varepsilon_{\mathrm{xx}}=\varepsilon_{\mathrm{yy}}\right)$ tensile strain of $0.4 \%$ is applied (see inset) to the 220 -nm-diameter CoFeB disk and then kept on. The gray dots, as well as the downward arrows mark the initial state $(t=0)$, highest transitional state $\left(t=t_{\mathrm{ts}}\right)$, and the equilibrium state $\left(t=t_{\mathrm{eq}}\right)$. $\mathbf{b}$, Corresponding spin structures at these three time stages showing a strain-mediated skyrmion creation. The interfacial DMl strength $D=0.75 \mathrm{~mJ} / \mathrm{m}^{2}$

of energy minimization, such that more Néel walls would form at equilibrium. When $D \geq 2.5 \mathrm{~mJ} / \mathrm{m}^{2}$, the initial QSD becomes unstable (Fig. 2b)

Second, the magnitude of critical strain generally decreases with increasing $D$, resulting from the reduced energy level of transitional states (Supplemental Fig. S7a). The latter is consistent with the reducing stability of the initial QSD at large $D$ (Fig. 2b). However, a plateau appears at intermediate $D$ values $(1.0-1.5 \mathrm{~mJ} /$ $\mathrm{m}^{2}$ ). This is because the number of transitional states increases with $D$ increasing from 1 to $1.5 \mathrm{~mJ} / \mathrm{m}^{2}$ (also see Fig. S7a), which offsets the reduction of critical strains.

The bottom panel of Fig. 5a shows the critical strain for skyrmion deletion as a function of $D$ under a constant diameter of $220 \mathrm{~nm}$. Different from the creation process, the magnitude of critical strain increases without a plateau as $D$ increases. This is due to the correspondingly higher energy level and the emergence of more transitional states (see Fig. S7b), both of which would increase critical strains. The higher energy level is an indicator of the enhanced stability of the skyrmion (Fig. 2b).

Having demonstrated that the $D$-dependence of critical strains is related to the $D$-dependent thermodynamic stabilities of the skyrmion and QSD, we further simulate the critical strains as a function of the diameter of the CoFeB nanodisk $\left(d_{\text {disk }}\right)$ under a constant $D$ of $0.75 \mathrm{~mJ} / \mathrm{m}^{2}$ (Fig. 5b). For the case of skyrmion creation, there are likewise two main messages. First, there exists a window of disk diameter $(140-480 \mathrm{~nm})$ only within which the skyrmion can be created from a QSD. When $d_{\text {disk }}$ is relatively small $(80-140 \mathrm{~nm})$, the skyrmion comes highly metastable (Fig. 2c) primarily due to the high stray field energy, and cannot be created by applying tensile $\varepsilon_{\text {app }}$. Instead, an in-plane vortex-like spin structure appears at equilibrium (see Supplemental Fig. S8), which
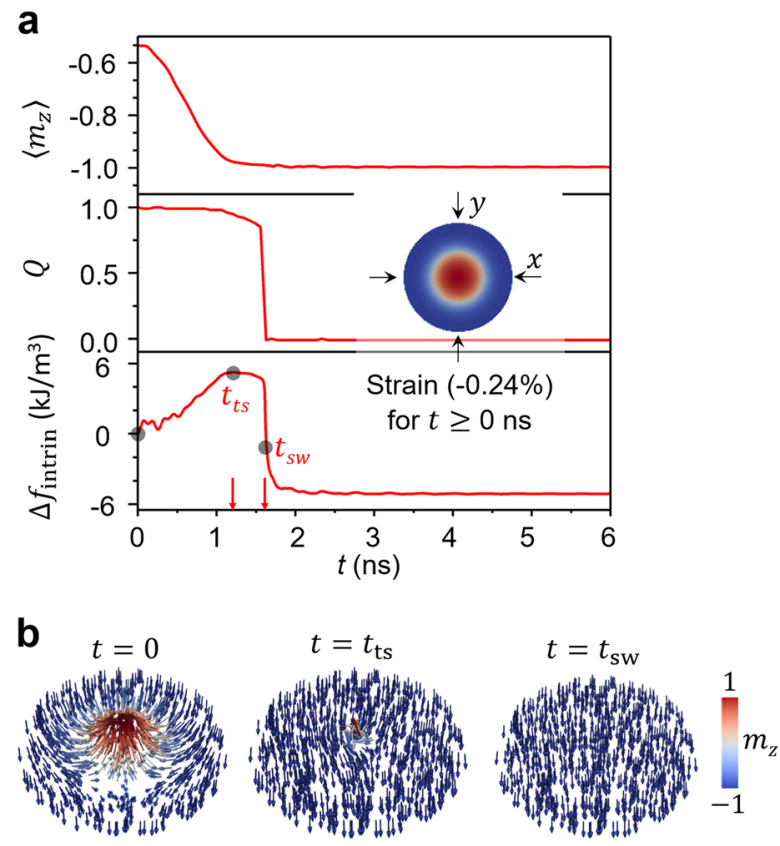

Fig. 4 Skyrmion deletion. a, Temporal evolution of the $m_{z}, Q$, and $\Delta f_{\text {intrin }}$ when a biaxial in-plane isotropic compressive strain of $-0.24 \%$ is applied (see inset) to the $220-\mathrm{nm}$-diameter CoFeB disk and then kept on. The gray dots, as well as the downward arrows mark the initial state $(t=0)$, highest transitional state $\left(t=t_{\mathrm{ts}}\right)$, and the completion of switching ( $t=t_{\mathrm{sw}}$, at which the skyrmion diameter reduces to zero). b. Corresponding spin structures at these three time stages showing a strain-mediated skyrmion deletion. The interfacial DMl strength $D=0.75 \mathrm{~mJ} / \mathrm{m}^{2}$

exhibits lower stray field energy than a skyrmion. When $d_{\text {disk }}$ is large (e.g., $720 \mathrm{~nm}$ ), a circular stripe domain appears at equilibrium (see also Fig. S8). Second, the magnitude of critical strain generally decreases due to the reduced stability of the initial QSD at large $d_{\text {disk. }}$ A plateau appears at intermediate disk diameters $(240-300 \mathrm{~nm})$, which likewise results from the competition between the lower energy level and the emergence of more transitional states during the kinetic process (see Supplemental Fig. S9a). The same principle can be applied to understand the increasing critical strain for skyrmion deletion without a plateau (see the bottom panel of Fig. 5b). In this case, the energy level becomes higher and more transitional states appears at large $d_{\text {disk }}$ (see Fig. S9b), both would increase the critical strain. Overall, it can be concluded that the critical strains for creating and deleting an isolated Néel skyrmion (Fig. 5) are primarily determined by the thermodynamic stabilities of the skyrmion and the QSD (Fig. 2).

The energy consumption $E_{\text {cons }}$ of a strain-mediated voltagecontrolled skyrmion switching is estimated as the minimum energy required to charge the piezoelectric capacitor, ${ }^{39}$ i.e., $E_{\text {cons }}$ $=0.5 P A U$. Here $P$ denotes the electric polarization of the piezoelectric under a driving voltage $U ; A$ is the area of the top electrode of the piezoelectric. Consider an epitaxial $\mathrm{Pb}\left(\mathrm{Zr}_{0.2} \mathrm{Ti}_{0.8}\right) \mathrm{O}_{3}$ (PZT) nanoisland as the piezoelectric. Our previous phase-field simulations ${ }^{40}$ have shown that a biaxial in-plane strain of larger than $1 \%$ can be achieved in a cuboid-shaped PZT nanoisland of $400 \mathrm{~nm}$ (length) $\times 400 \mathrm{~nm}$ (width) $\times 100 \mathrm{~nm}$ (thickness) through voltage-induced $90^{\circ}$ ferroelectric domain switching. Notably, the bipolar biaxial in-plane strain required for skyrmion creation and deletion can be accessed by engineering the size/shape of the PZT nanoisland and its misfit strain with the substrate, under a driving voltage of $\sim 1 \mathrm{~V}$. ${ }^{41}$ Taking $P=0.75 \mathrm{C} / \mathrm{m}^{2}$ for the PZT nanoisland ${ }^{42}$ and $U=1 \mathrm{~V}, E_{\text {cons }} \approx 6 \mathrm{fJ}$ for $A \approx 0.015 \mu \mathrm{m}^{2}$ (i.e., the surface area of a 140-nm-diameter CoFeB disk, which is used as a top electrode of the PZT nanoisland). $E_{\text {cons }}$ can be reduced to $\sim 0.5 \mathrm{fJ}$ if using a 

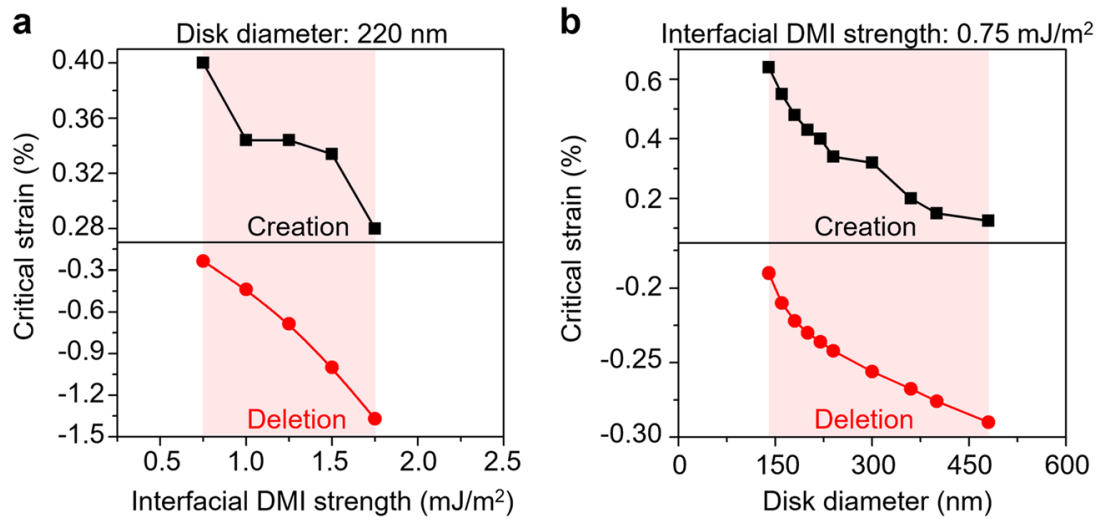

Fig. 5 On critical strains. Influence of $\mathbf{a}$, the interfacial DMl strength $D, \mathbf{b}$, the disk diameter on the critical (minimum) strains for skyrmion creation and deletion. A constant disk diameter of $220 \mathrm{~nm}$ is used for cases in a, a constant $D$ of $0.75 \mathrm{~mJ} / \mathrm{m}^{2}$ is used for cases in $\mathbf{b}$

separate 40-nm-diameter electrode $\operatorname{dot}^{43}\left(A \approx 0.0013 \mu \mathrm{m}^{2}\right)$. Notably, the estimated $0.5 \mathrm{fJ}$ is about 5 orders of magnitude smaller than that in a spin-transfer-torque-mediated currentcontrolled skyrmion switching (see Supplemental Fig. S10-11 and Note S2).

In summary, we have computationally demonstrated a repeatable switching (creation and deletion) of an isolated Néel skyrmion purely driven by voltage-induced strains transmitted from a juxtaposed piezoelectric. An ultralow-energy consumption of $\sim 0.5 \mathrm{fJ}$ per switching is envisaged. Unlike existing reports of voltage $^{21,22}$ or stress ${ }^{25,30}$ controlled skyrmion switching in magnetic bulk crystals or thin films, the present scheme demonstrated in a 3D geometrically confined nanomagnet obviates the need of using a magnetic field. Although a magnetic ultrathin nanodisk with interfacial DMI is used for demonstration, the computational model established through this work can be adapted to model strain-mediated skyrmion switching in magnetic bulk crystals/thin-films/nanostructures that exhibit bulk-type DMI. Our findings are expected to stimulate experimental efforts into strain-mediated voltage control of skyrmions and other chiral spin structures for ultralow-energy spintronic devices.

\section{METHODS}

In our phase-field model, the spin structure is described by the spatial distributions of local $\mathbf{M}=M_{s}\left(m_{x}, m_{y}, m_{z}\right)$, where $m_{i}(i=x, y, z)$ the normalized local magnetization along a Cartesian axis. The total magnetic free energy $F_{\text {tot }}(\mathbf{m})$ of a magnetic ultrathin nanostructure with DMI can be expressed as,

$F_{\text {tot }}=\int\left(f_{\text {anis }}+f_{\text {exch }}+f_{\text {DMI }}+f_{\text {stray }}+f_{\text {elast }}\right) d V$,

where the terms in the integrand denote the volumetric densities $\left(\mathrm{J} / \mathrm{m}^{3}\right)$ of perpendicular magnetic anisotropy (arising from the interface), Heisenberg-type symmetric exchange energy, Dyzaloshinskii-Moriya (DM)-type antisymmetric exchange energy, stray field energy, and elastic energy, respectively. The average intrinsic energy density $f_{\text {intrin }}$ is defined as the sum of the volume average of the $f_{\text {anis, }}, f_{\text {exch, }}, f_{\mathrm{DMl}}$, and $f_{\text {stray }}$.

The expression of $f_{\text {anis }}$ has the typical form of uniaxial magnetic anisotropy along the $z$ axis,

$f_{\text {anis }}=K_{1}\left(1-m_{\mathrm{z}}^{2}\right)+K_{2}\left(1-m_{\mathrm{z}}^{2}\right)^{2}$

where $K_{1}$ and $K_{2}$ are coefficients of perpendicular magnetic anisotropy, and $m_{z}$ is the normalized magnetization along the $z$ axis. Here the perpendicular magnetic anisotropy mainly arise from the magnetic interface anisotropy ${ }^{44} f_{\text {inter }}=-\left(K_{\text {inter }} / d\right) m_{z}^{2}$, where $K_{\text {inter }} \approx K_{1} d \approx 1.08 \mathrm{~mJ} /$ $\mathrm{m}^{2}$. The Heisenberg-type symmetric exchange energy density is given by $f_{\text {exch }}=A_{\text {ex }}(\nabla \mathbf{m})^{2}$
In an ultrathin magnet where the DMI arises from the interface with its adjacent heavy metals, the interfacial DMI free energy $f_{\mathrm{DMI}}$ is given as, ${ }^{45}$

$f_{\text {DMI }}=D\left(m_{z} \frac{\partial m_{x}}{\partial x}-m_{x} \frac{\partial m_{z}}{\partial x}+m_{z} \frac{\partial m_{y}}{\partial y}-m_{y} \frac{\partial m_{z}}{\partial y}\right)$.

When $D>0$, minimizing $f_{\mathrm{DMI}}^{\mathrm{i}}$ yields a positive $\frac{\partial m_{z}}{\partial x}$ or $\frac{\partial m_{z}}{\partial x}$ in the propagating direction of the cycloidal spin spiral (see Fig. 1c). Note that $D$ is proportional to $\frac{\left|\mathbf{D}_{12}\right|}{a d}$, ${ }^{6}$ where $\mathbf{D}_{12}$ describes the atomistic DMI (denoted as $\mathbf{H}_{\mathrm{DM}}$ ) between two neighboring atomic spins $S_{1}$ and $S_{2}$ through $\mathbf{H}_{\mathrm{DM}}=$ $-\mathbf{D}_{12}\left(\mathbf{S}_{1} \times \mathbf{S}_{2}\right), a$ is the lattice parameter of the ultrathin magnet. For an amorphous or polycrystalline magnetic disk with $\infty \mathrm{m}$ symmetry, $\mathbf{D}_{12}$ is always parallel to the magnet/heavy-metal interface (see Fig. 1a). Note that $\mathbf{D}_{12}$ may change once the applied strain modifies the lattice parameter $a$. Due to a lack of available data of strain-modulated $D$ in magnetic ultrathin nanostructures, we discussed the influence of $D$ on the thermodynamic stability of a skyrmion (Fig. 2) and the critical strains for skyrmion creation/ deletion (Fig. 5a).

In a confined magnetic system with interfacical DMI, using variational calculation, ${ }^{46}$ the variations of the exchange energy and interfacial DMI energy are written as

$\delta\left(\int f_{\text {exch }} d V\right)=2 A_{\text {ex }} \int_{\partial V} \frac{\partial \mathbf{m}}{\partial \mathbf{n}} \cdot \delta \mathbf{m} d S-2 A_{\mathrm{ex}} \int\left(\nabla^{2} \mathbf{m}\right) \cdot \delta \mathbf{m} d V$

$\delta\left(f_{\mathrm{DMI}} d V\right)=-D \int_{\partial V}((\mathbf{n} \times z) \times \mathbf{m}) \cdot \delta \mathbf{m} d S+2 D\left((\nabla \cdot \mathbf{m}) z-\nabla m_{z}\right) \cdot \delta \mathbf{m} d V$

where $\partial V$ denotes the surface of the magnet, $\mathbf{n}$ is the outward-pointing unit normal of the surface, and $d S$ is the surface element. The variations of the free energies contain the surface terms and the volume terms (the second integrals on the right). Minimizing the combined surface terms of the variations of exchange energy and the interfacial DMI energy (i.e., the first integrals on the right of Eqs. 5-6), i.e., $\delta F_{\text {Surface }}=2 A_{\mathrm{ex}} \int_{\partial V}\left(\frac{\partial \mathbf{m}}{\partial \mathbf{n}}-\frac{D}{2 A_{\mathrm{ex}}}(\mathbf{n} \times z) \times \mathbf{m}\right) \cdot \delta \mathbf{m} d S=0$, yields the boundary condition,

$\frac{d \mathbf{m}}{d \mathbf{n}}=\frac{D}{2 A_{\mathrm{ex}}}(\mathbf{n} \times z) \times \mathbf{m}$.

The stray field energy density $f_{\text {stray }}=-0.5 \mu_{0} M_{s} \mathbf{H}_{\mathrm{d}} \cdot \mathbf{m}$, where the stray field $\mathbf{H}_{\mathrm{d}}$ is obtained by solving the magnetostatic equilibrium equation $\nabla \cdot \mu_{0}\left(\mathbf{H}_{\mathrm{d}}+M_{s} \mathbf{m}\right)=0$ under a finite-size boundary condition based on the convolution theorem evaluated using the Fast Fourier Transform. ${ }^{47}$

The elastic energy density $f_{\text {elast }}=0.5\left(\boldsymbol{\varepsilon}^{\text {tot }}-\boldsymbol{\varepsilon}^{0}\right) \cdot \mathbf{c} \cdot\left(\boldsymbol{\varepsilon}^{\text {tot }}-\boldsymbol{\varepsilon}^{0}\right)$, where $\mathbf{c}$ denotes the elastic stiffness tensor. The stress-free strain $\varepsilon_{i i}^{0}=$ $\frac{3}{2} \lambda_{100}\left(m_{i}^{2}-\frac{1}{3}\right)$ and $\varepsilon_{i k}^{0}=\frac{3}{2} \lambda_{111} m_{i} m_{k}$ for $i, k=x, y, z$. The total strain $\boldsymbol{\varepsilon}^{\text {tot }}=\bar{\varepsilon}+\boldsymbol{\varepsilon}^{\text {het }}$. The first term $\overline{\boldsymbol{\varepsilon}}$ is the average strain over the three-phase simulation system (including the nanodisk, piezoelectric, and vacuum, see Supplemental Fig. S12), which equals the homogeneous strain on the bottom plane of the piezoelectric layer. The second term $\varepsilon^{\text {het }}$ describes the local variations of strains that are related to both the size/geometry of the magnetic nanostructure and the spatially varying magnetizations, and is obtained by solving the elastic equilibrium equation $\nabla \cdot\left(\mathbf{c}\left(\boldsymbol{\varepsilon}^{\text {tot }}-\boldsymbol{\varepsilon}^{0}\right)\right)=0$ 
using the Fourier-spectral iteration method. ${ }^{48}$ Since $\boldsymbol{\varepsilon}^{0}$ depends on the local magnetization $\mathbf{m}$, a two-way coupling between the $\mathbf{m}$ and the $\boldsymbol{\varepsilon}^{\text {het }}$ is established. Similar approach has been adopted by other phase-field models ${ }^{49}$ or finite-element models ${ }^{50}$ in order to achieve such full coupling between local magnetization dynamics and elasticity. A fixed displacement boundary condition (corresponding to the applied biaxial in-plane strain $\boldsymbol{\varepsilon}_{\text {app }}$ ) is applied on the bottom plane of the piezoelectric in the simulation system (see Fig. S12), while a stress-free boundary condition at the top surface of the magnetic disk; see details in ref. ${ }^{51}$ The stress-free boundary conditions at the lateral surfaces of the island are automatically considered by setting the elastic stiffness tensor of the surrounding vacuum as zero.

It is worth pointing out that the equilibrium average strain in the nanodisk is slightly smaller than the applied strain $\boldsymbol{\varepsilon}_{\text {app }}$ due to strain relaxation near the lateral surfaces of the nanodisk. For example, under a biaxial in-plane isotropic $\boldsymbol{\varepsilon}_{\mathrm{app}}$ of $0.4 \%$, the average strain in a $220-\mathrm{nm}$ diameter nanodisk is $0.381 \%$. The equilibrium strain distribution simulated using our in-house elastic solver described above is well consistent with that obtained from finite-element analysis in COMSOL Multiphysics (see Supplemental Fig. S13).

The variational derivative of the $F_{\text {tot }}$ with respect to the local magnetization yields the expression of effective magnetic field, i.e., $\mathbf{H}_{\text {eff }}=-\frac{1}{\mu_{0} M_{s}} \frac{\delta F_{\text {tot }}}{\delta \mathbf{m}}$, which is the driving force for the evolution of local magnetization $\mathbf{m}$ in the framework of Landau-Lifshitz-Gilbert (LLG) type dynamics. The classical LLG equation reads as,

$\frac{\partial \mathbf{m}}{\partial t}=-\frac{\gamma_{0}}{1+a^{2}}\left[\left(\mathbf{m} \times \mathbf{H}_{\text {eff }}\right)+a \mathbf{m} \times\left(\mathbf{m} \times \mathbf{H}_{\text {eff }}\right)\right]$,

where $\gamma_{0}$ is the gyromagnetic ratio and $a$ the Gilbert damping coefficient. Eq. (8) was solved using the classical Runge-Kutta method with a dimensionless discretized time step $\Delta t^{*}=\frac{\gamma_{0} M_{s}}{1+\alpha^{2}} \Delta t=0.01$. Our testing shows that the use of a smaller reduced time step of 0.005 yields almost the same result. The equilibrium state (e.g., $t=t_{\text {eq, }}$ see Fig. 3 ) is considered to be reached when $\left|\Delta\left\langle m_{z}\right\rangle / \Delta t^{*}\right|<10^{-4}$, where $\Delta\left\langle m_{z}\right\rangle$ denotes the change in the volume average of normalized perpendicular magnetization $m_{z}$ per time step $\Delta t^{*}$. The simulations were performed in three-dimensional discretized cells of $n_{x} \Delta x \times n_{y} \Delta y \times n_{z} \Delta z$, where $n_{i}$ and $\Delta i(i=x, y, z)$ indicate the number of cells and corresponding cell size along a Cartesian axis. Specifically, we use a constant $n_{z}=17$ where the bottom 11 layers of grids describe the elastically stiff piezoelectric layer (meaning that the piezoelectric has a large Young's modulus), the middle 2 layers describes the magnetic ultrathin nanostructure including its lateral surrounding vacuum space, and the top 4 layers describes the space on top of the magnet. $\Delta z$ is set as $0.55 \mathrm{~nm}$ to describe a $1.1-\mathrm{nm}$-thick magnetic disk. Various disk diameters were described by varying $n_{x}$ and $n_{y}$ under a constant in-plane cell size $\Delta x=\Delta y=1 \mathrm{~nm}$. In most cases, using a smaller in-plane cell of $0.5 \mathrm{~nm}$ yields almost the same results. However, when either the interfacial DMI strength $D$ or the disk diameter is relatively small (e.g, see blue circles in Fig. $2 b, c$ where the skyrmion has a notably higher energy than the other spin structures), only the use of smaller in-plane cells (e.g., $0.5 \mathrm{~nm}$ ) allows us to access these highly metastable skyrmions. A similar case has been reported in previous micromagnetic simulations. ${ }^{6}$

The materials parameters for ultrathin $\mathrm{CO}_{20} \mathrm{Fe}_{60} \mathrm{~B}_{20}$ used in simulations are listed as follows: $K_{1}=0.978 \mathrm{~mJ} / \mathrm{m}^{3}, K_{2}=11.08 \mathrm{~kJ} / \mathrm{m}^{3}, M_{s}=1.25 \mathrm{MA} / \mathrm{m}$, $a=0.04, \quad \lambda_{100}=\lambda_{111}=\lambda_{s}=3.7 \times 10^{-5} \quad$ (ref. ${ }^{52}$ ); $\gamma_{0}=2.2 \times 10^{5} \mathrm{~m} \mathrm{~A}^{-1} \cdot \mathrm{s}^{-1}$ (ref. ${ }^{53}$ ); $A_{\mathrm{ex}}=1.9 \times 10^{-11} \mathrm{~J} / \mathrm{m}$ for $\mathrm{Co}_{20} \mathrm{Fe}_{60} \mathrm{~B}_{20}$ (CoFeB) nanoislands with a thickness range of $0.9-1.3 \mathrm{~nm}$ (ref. ${ }^{54}$ ); $c_{11}=218.1 \mathrm{GPa}, c_{12}=93.46 \mathrm{GPa}$, which were calculated based on a Young's modulus of $162 \mathrm{GPa}\left(\right.$ ref. $\left.^{55}\right)$ and a Poisson's ratio of 0.3 (ref. ${ }^{56}$ ); In the present simulations, the elastic stiffness tensor of the piezoelectric is set as the same as that of the magnetic island, i.e., $\boldsymbol{c}_{\mathrm{p}}=\mathbf{c}_{\text {island }}$ for simplicity. Nevertheless, our tests show that the equilibrium strain state in the magnetic nanodisk would not have an appreciable difference if the piezoelectric layer has a relatively high Young's modulus (see Supplemental Table S1 and a brief discussion therein).

\section{DATA AVAILABILITY}

The data that support the plots presented in this paper and its supplemental information files and other findings of this study are available from the corresponding authors upon reasonable request.

\section{ACKNOWLEDGEMENTS}

The work is supported by the Army Research Office under grant number W911NF-171-0462 and the US National Science Foundation under the DMREF program DMR1629270. J.-M.H. also acknowledge a start-up fund from the University of Wisconsin-Madison.

\section{AUTHOR CONTRIBUTIONS}

J.-M.H. initiated the idea, designed the simulation plan and structure of the paper, and performed the majority part of the simulations. T.Y. developed the parallel computer codes and helped perform the simulations. L.-Q.C. supervised the study. J.M.H. wrote the paper with feedback from T.Y. and L.-Q.C. J.-M.H., and T.Y. contributed equally to this work.

\section{ADDITIONAL INFORMATION}

Supplementary information accompanies the paper on the npj Computational Materials website (https://doi.org/10.1038/s41524-018-0119-2).

Competing interests: The authors declare no competing interests.

Publisher's note: Springer Nature remains neutral with regard to jurisdictional claims in published maps and institutional affiliations.

\section{REFERENCES}

1. Nagaosa, N. \& Tokura, Y. Topological properties and dynamics of magnetic skyrmions. Nat. Nanotechnol. 8, 899-911 (2013).

2. Wiesendanger, R. Nanoscale magnetic skyrmions in metallic films and multilayers: a new twist for spintronics. Nat. Rev. Mater. 1, 16044 (2016).

3. Fert, A., Reyren, N. \& Cros, V. Magnetic skyrmions: advances in physics and potential applications. Nat. Rev. Mater. 2, 17031 (2017).

4. Jiang, W. J. et al. Skyrmions in magnetic multilayers. Phys. Rep. 704, 1-49 (2017).

5. Wataru, K. et al. Memory functions of magnetic skyrmions. Jpn. J. Appl. Phys. 54, 053001 (2015)

6. Sampaio, J., Cros, V., Rohart, S., Thiaville, A. \& Fert, A. Nucleation, stability and current-induced motion of isolated magnetic skyrmions in nanostructures. Nat. Nanotechnol. 8, 839-844 (2013).

7. Romming, N. et al. Writing and Deleting Single Magnetic Skyrmions. Science 341, 636-639 (2013).

8. Flovik, V., Qaiumzadeh, A., Nandy, A. K., Heo, C. \& Rasing, T. Generation of single skyrmions by picosecond magnetic field pulses. Phys. Rev. B 96, 140411 (2017).

9. Jonietz, F. et al. Spin Transfer Torques in MnSi at Ultralow Current Densities. Science 330, 1648-1651 (2010).

10. $\mathrm{Yu}, \mathrm{X}$. Z. et al. Skyrmion flow near room temperature in an ultralow current density. Nat. Commun. 3, 988 (2012).

11. Iwasaki, J., Mochizuki, M. \& Nagaosa, N. Current-induced skyrmion dynamics in constricted geometries. Nat. Nanotechnol. 8, 742-747 (2013).

12. Jiang, W. et al. Blowing magnetic skyrmion bubbles. Science 349, 283-286 (2015).

13. Woo, S. et al. Observation of room-temperature magnetic skyrmions and their current-driven dynamics in ultrathin metallic ferromagnets. Nat. Mater. 15, 501-506 (2016).

14. Yu, G. et al. Room-Temperature Creation and Spin-Orbit Torque Manipulation of Skyrmions in Thin Films with Engineered Asymmetry. Nano Lett. 16, 1981-1988 (2016).

15. Legrand, W. et al. Room-Temperature Current-Induced Generation and Motion of sub-100 nm Skyrmions. Nano Lett. 17, 2703-2712 (2017).

16. Büttner, F. et al. Field-free deterministic ultrafast creation of magnetic skyrmions by spin-orbit torques. Nat. Nanotechnol. 12, 1040-1044 (2017).

17. Zhou, Y. \& Ezawa, M. A reversible conversion between a skyrmion and a domainwall pair in a junction geometry. Nat. Commun. 5, 4652 (2014).

18. Finazzi, M. et al. Laser-Induced Magnetic Nanostructures with Tunable Topological Properties. Phys. Rev. Lett. 110, 177205 (2013).

19. Koshibae, W. \& Nagaosa, N. Creation of skyrmions and antiskyrmions by local heating. Nat. Commun. 5, 5148 (2014).

20. White, J. S. et al. Electric-Field-Induced Skyrmion Distortion and Giant Lattice Rotation in the Magnetoelectric Insulator $\mathrm{Cu}_{2} \mathrm{OSeO}_{3}$. Phys. Rev. Lett. 113, 107203 (2014).

21. Hsu, P.-J. et al. Electric-field-driven switching of individual magnetic skyrmions. Nat. Nanotechnol. 12, 123-126 (2016).

22. Schott, M. et al. The Skyrmion Switch: Turning Magnetic Skyrmion Bubbles on and off with an Electric Field. Nano Lett. 17, 3006-3012 (2017). 
23. Srivastava, T. et al. Large-voltage tuning of Dzyaloshinskii-Moriya Interactions: A route toward dynamic control of skyrmion chirality. Nano Lett. 18, 4871-4877 (2018).

24. Butenko, A. B., Leonov, A. A., Rößler, U. K. \& Bogdanov, A. N. Stabilization of skyrmion textures by uniaxial distortions in noncentrosymmetric cubic helimagnets. Phys. Rev. B 82, 052403 (2010).

25. Nii, Y. et al. Uniaxial stress control of skyrmion phase. Nat. Commun. 6, 8539 (2015).

26. Shibata, K. et al. Large anisotropic deformation of skyrmions in strained crystal. Nat. Nanotechnol. 10, 589-592 (2015).

27. Liu, Y. et al. Chopping skyrmions from magnetic chiral domains with uniaxial stress in magnetic nanowire. Appl. Phys. Lett. 111, 022406 (2017).

28. Li, Z. et al. Strain-controlled skyrmion creation and propagation in ferroelectric/ ferromagnetic hybrid wires. J. Magn. Magn. Mater. 455, 19-24 (2018).

29. Seki, S. et al. Stabilization of magnetic skyrmions by uniaxial tensile strain. Phys. Rev. B 96, 220404 (2017).

30. Wang, J., Shi, Y. \& Kamlah, M. Uniaxial strain modulation of the skyrmion phase transition in ferromagnetic thin films. Phys. Rev. B 97, 024429 (2018).

31. Boulle, O. et al. Room-temperature chiral magnetic skyrmions in ultrathin magnetic nanostructures. Nat. Nanotechnol. 11, 449-454 (2016).

32. Zheng, F. et al. Direct imaging of a zero-field target skyrmion and its polarity switch in a chiral magnetic nanodisk. Phys. Rev. Lett. 119, 197205 (2017).

33. Thiele, C., Dörr, K., Bilani, O., Rödel, J. \& Schultz, L. Influence of strain on the magnetization and magnetoelectric effect in $\mathrm{La}_{0.7} \mathrm{~A}_{0.3} \mathrm{MnO}_{3} / \mathrm{PMN}-\mathrm{PT}(001)(\mathrm{A}=\mathrm{Sr}$ Ca). Phys. Rev. B 75, 054408 (2007).

34. Eerenstein, W., Wiora, M., Prieto, J. L., Scott, J. F. \& Mathur, N. D. Giant sharp and persistent converse magnetoelectric effects in multiferroic epitaxial heterostructures. Nat. Mater. 6, 348-351 (2007).

35. Cherifi, R. O. et al. Electric-field control of magnetic order above room temperature. Nat. Mater. 13, 345-351 (2014).

36. Ghidini, M. et al. Perpendicular Local Magnetization Under Voltage Control in Ni Films on Ferroelectric $\mathrm{BaTiO}_{3}$ Substrates. Adv. Mater. 27, 1460-1465 (2015)

37. Hu, J.-M., Chen, L.-Q. \& Nan, C.-W. Multiferroic Heterostructures Integrating Fer roelectric and Magnetic Materials. Adv. Mater. 28, 15-39 (2016).

38. Rohart, S. \& Thiaville, A. Skyrmion confinement in ultrathin film nanostructures in the presence of Dzyaloshinskii-Moriya interaction. Phys. Rev. B 88, 184422 (2013).

39. Hu, J.-M., Li, Z., Chen, L.-Q. \& Nan, C.-W. High-density magnetoresistive random access memory operating at ultralow voltage at room temperature. Nat. Commun. 2, 553 (2011)

40. $\mathrm{Hu}$, J.-M. et al. Purely electric-field-driven perpendicular magnetization reversal. Nano Lett. 15, 616-622 (2015).

41. Zhang, J. X., Sheng, G. \& Chen, L. Q. Large electric field induced strains in ferroelectric islands. Appl. Phys. Lett. 96, 132901 (2010).

42. Nagarajan, V. et al. Dynamics of ferroelastic domains in ferroelectric thin films. Nat. Mater. 2, 43-47 (2003)

43. Ikeda, S. et al. A perpendicular-anisotropy $\mathrm{CoFeB}-\mathrm{MgO}$ magnetic tunnel junction. Nat. Mater. 9, 721-724 (2010)
44. Hu, J.-M., Nan, C.-W. \& Chen, L.-Q. Size-dependent electric voltage controlled magnetic anisotropy in multiferroic heterostructures: Interface-charge and strain comediated magnetoelectric coupling. Phys. Rev. B 83, 134408 (2011).

45. Bogdanov, A. N. \& Rößler, U. K. Chiral Symmetry Breaking in Magnetic Thin Films and Multilayers. Phys. Rev. Lett. 87, 037203 (2001).

46. Miltat, J. in Applied Magnetism (eds Richard Gerber, C. D. Wright, \& G. Asti) 221-308 (Springer Netherlands, 1994).

47. Fabian, K. et al. Three-dimensional micromagnetic calculations for magnetite using FFT. Geophys. J. Int. 124, 89-104 (1996).

48. Hu, S. Y. \& Chen, L. Q. A phase-field model for evolving microstructures with strong elastic inhomogeneity. Acta Mater. 49, 1879-1890 (2001).

49. Yi, M., Xu, B.-X. \& Shen, Z. $180^{\circ}$ magnetization switching in nanocylinders by a mechanical strain. Extrem. Mech. Lett. 3, 66-71 (2015).

50. Liang, C.-Y. et al. Modeling of magnetoelastic nanostructures with a fully coupled mechanical-micromagnetic model. Nanotechnology 25, 435701 (2014)

51. Li, Y. L., Hu, S. Y., Liu, Z. K. \& Chen, L. Q. Effect of substrate constraint on the stability and evolution of ferroelectric domain structures in thin films. Acta Mater. 50, 395-411 (2002).

52. $\mathrm{Yu}, \mathrm{G}$. et al. Strain-induced modulation of perpendicular magnetic anisotropy in $\mathrm{Ta} / \mathrm{CoFeB} / \mathrm{MgO}$ structures investigated by ferromagnetic resonance. Appl. Phys. Lett. 106, 072402 (2015).

53. Hu, J.-M., Yang, T. N., Chen, L. Q. \& Nan, C. W. Voltage-driven perpendicular magnetic domain switching in multiferroic nanoislands. J. Appl. Phys. 113, 194301 (2013).

54. Sato, H. et al. CoFeB Thickness Dependence of Thermal Stability Factor in CoFeB/ MgO Perpendicular Magnetic Tunnel Junctions. IEEE Magn. Lett. 3, 3000204 (2012).

55. Hindmarch, A. T., Rushforth, A. W., Campion, R. P., Marrows, C. H. \& Gallagher, B. L. Origin of in-plane uniaxial magnetic anisotropy in CoFeB amorphous ferromagnetic thin films. Phys. Rev. B 83, 212404 (2011).

56. Wang, D., Nordman, C., Qian, Z., Daughton, J. M. \& Myers, J. Magnetostriction effect of amorphous CoFeB thin films and application in spin-dependent tunnel junctions. J. Appl. Phys. 97, 10 C906 (2005).

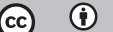

Open Access This article is licensed under a Creative Commons Attribution 4.0 International License, which permits use, sharing, adaptation, distribution and reproduction in any medium or format, as long as you give appropriate credit to the original author(s) and the source, provide a link to the Creative Commons license, and indicate if changes were made. The images or other third party material in this article are included in the article's Creative Commons license, unless indicated otherwise in a credit line to the material. If material is not included in the article's Creative Commons license and your intended use is not permitted by statutory regulation or exceeds the permitted use, you will need to obtain permission directly from the copyright holder. To view a copy of this license, visit http://creativecommons. org/licenses/by/4.0/.

(c) The Author(s) 2018 